\title{
Cluster de Sintomas e o Impacto na Qualidade de Saúde Global de Pacientes com Câncer Avançado
}

https://doi.org/10.32635/2176-9745.RBC.2021v67n3.1190

\author{
Symptom Cluster and the Impact on the Global Health Quality of Patients with Advanced Cancer \\ Grupo de Síntomas y el Impacto en la Calidad Global de la Salud de los Pacientes con Cáncer Avanzado
}

Iza Rodrigues Mello'; Noélly Maura de Jesus Guimarães²; Luana Silva Monteiro³; Gunnar de Cunto Carelli Taets ${ }^{4}$

RESUMO

Introduçáo: Os pacientes com câncer podem apresentar múltiplos sintomas que se inter-relacionam, formando os chamados clusters ou agrupamentos de sintomas. Objetivo: Avaliar a relação entre um cluster de sintomas e a qualidade de saúde global de pacientes com câncer avançado. Método: Estudo analítico, transversal, com 146 pacientes. Foram utilizados instrumentos de caracterização sociodemográfica e de avaliação de qualidade de vida. Para análise dos dados, aplicaram-se os testes Kolmogorov-Smirnov, Mann-Whitney e Kruskal-Wallis. A coleta de dados foi realizada entre julho de 2019 e fevereiro de 2020 e utilizado o questionário 30-item European Organization for Research and Treatment of Cancer Core Quality of Life Questionnaire (EORTC QLQ-C30), versáo 3. Resultados: A correlaçáo de Spearman mostrou-se positiva entre o escore final da escala de sintomas e o escore final da avaliação global da saúde $(\rho=0,605 ; p<0,001)$. Além disso, observou-se que o incremento de 1 ponto na pergunta: "Precisou descansar?" associou-se com aumento de 5,87 pontos no escore da qualidade da saúde global ( $<<0,01)$; para a pergunta "Sentiu-se cansado/a?", 6,14 pontos ( $\mathrm{p}<0,01)$; e "Teve falta de ar?", 5,08 pontos $(\mathrm{p}<0,01)$. Conclusáo: Existe uma correlação positiva entre o cluster de sintomas composto por dor, fadiga, dispneia, náusea, vômito, insônia, inapetência, constipação, diarreia e a qualidade de saúde global de pacientes com câncer.

Palavras-chave: Neoplasias; Qualidade de Vida; Perfil de Impacto da Doença; Sinais e Sintomas.

\section{ABSTRACT}

Introduction: Patients with cancer can present multiple symptoms that are interrelated, forming the so-called clusters or groups of symptoms. Objective: To evaluate the relationship between a cluster of symptoms and the global quality of life of patients with advanced cancer. Method: Analytical, cross-sectional study with 146 patients. Sociodemographic characterization and quality of life assessment instruments were used. For data analysis, the Kolmogorov-Smirnov, Mann-Whitney, and Kruskal-Wallis tests were applied. Data collection was carried out between July 2019 and February 2020 and the European Organization for Research and Treatment of Cancer Core Quality of Life Questionnaire (EORTC QLQ-C30), version 3, was adopted. Results: Spearman's correlation was positive between the final score of the symptom scale and the final score of the global health assessment $(\rho=0.605 ; p<0.001)$. In addition, it was observed that the increase of 1 point in the question "Did you need to rest?" was associated with an increase of 5.87 points in the global health quality score $(\mathrm{p}<0.01)$, for the question "Did you feel tired?", 6.14 points $(\mathrm{p}<0.01)$ and "Did you have shortness of breath?", 5.08 points $(\mathrm{p}<0.01)$. Conclusion: There is a positive correlation between the cluster of symptoms formed by pain, fatigue, dyspnea, nausea, vomiting, insomnia, lack of appetite, constipation, diarrhea and the overall health quality of patients with cancer.

Key word: Neoplasms; Quality of Life; Sickness Impact Profile; Signs and Symptoms.

\section{RESUMEN}

Introducción: Los pacientes con cáncer pueden presentar múltiples síntomas que están interrelacionados, formando los llamados grupos o grupos de síntomas. Objetivo: Evaluar la relación entre uno grupo de síntomas y la calidad de vida general de los pacientes con cáncer avanzado. Método: Estudio analítico, transversal, con 146 pacientes. Se utilizaron instrumentos de caracterización sociodemográfica y de evaluación de la calidad de vida. Para el análisis de datos, se aplicaron las pruebas de Kolmogorov-Smirnov, Mann-Whitney y Kruskal-Wallis. La recolección de datos se llevó a cabo entre julio de 2019 y febrero de 2020 y se utilizó el cuestionario de calidad de vida básica de la Organización Europea para la Investigación y el Tratamiento del Cáncer (EORTC QLQ-C30), versión 3. Resultados: La correlación de Spearman fue positiva entre el puntaje final de la escala de síntomas y el puntaje final de la evaluación de salud global $(\rho=0,605 ; p<0,001)$. Además, se observó que el aumento de 1 punto en la pregunta “¿Necesitabas descansar?” se asoció con un aumento de 5,87 puntos en el puntaje de calidad de salud global $(\mathrm{p}<0,01)$; para la pregunta "¿Se sintió cansado?”, 6,14 puntos ( $\mathrm{p}<0,01) ; \mathrm{y}$ “'Tuvo dificultad para respirar?”, 5,08 puntos $(\mathrm{p}<0,01)$. Conclusión: Existe una correlación positiva entre el conjunto de síntomas compuestos por dolor, fatiga, disnea, náuseas, vómitos, insomnio, falta de apetito, estreńimiento, diarrea y la calidad de salud general de los pacientes con cáncer.

Palabra clave: Neoplasias; Calidad de Vida; Perfil de Impacto de Enfermedad; Signos y Síntomas.

\footnotetext{
1-4Universidade Federal do Rio de Janeiro. Macaé (RJ), Brasil.

'E-mail: izarodriguesmello@gmail.com. Orcid iD: https://orcid.org/0000-0003-2632-9229

${ }^{2}$ E-mail: noellyguimaraes@gmail.com. Orcid iD: https://orcid.org/0000-0003-4986-5833

${ }^{3}$ E-mail: luananutrir@gmail.com. Orcid iD: https://orcid.org/0000-0003-3599-6947

${ }^{4}$ E-mail: masterufrj@gmail.com. Orcid iD: https://orcid.org/0000-0003-4427-7864

Endereço para correspondência: Iza Rodrigues Mello. Rua Valdelina Viana Santana C, 19 - Visconde de Araújo. Macaé (RJ), Brasil. CEP 27940-450.

E-mail: izarodriguesmello@gmail.com
} 


\section{INTRODUÇÃO}

O câncer está entre as quatro principais causas de morte prematura (antes dos 70 anos de idade) na maioria dos países, tornando-se o principal problema de saúde pública no mundo. Segundo a mais recente estimativa mundial, ocorreram no mundo 18 milhóes de casos novos de câncer (17 milhôes sem contar os casos de câncer de pele náo melanoma) e 9,6 milhóes de óbitos (9,5 milhões, excluindo os cânceres de pele não melanoma) ${ }^{1}$.

Para cada ano do triênio 2020-2022, a estimativa do Instituto Nacional de Câncer José Alencar Gomes da Silva (INCA) aponta para 625 mil casos novos de câncer (450 mil, excluindo os casos de câncer de pele não melanoma) no Brasil. $\mathrm{O}$ câncer de pele não melanoma será o mais incidente (177 mil), seguido pelos cânceres de mama e próstata (66 mil cada), cólon e reto (41 mil), pulmão (30 mil) e estômago $(21 \mathrm{mil})^{2}$. Os pacientes com câncer podem apresentar múltiplos sintomas que se inter-relacionam, formando os chamados clusters, ou agrupamentos de sintomas ${ }^{3}$. Esses sintomas não necessariamente compartilham a mesma etiologia e podem ser definidos também quando dois ou mais sintomas estão relacionados entre si e ocorrem em conjunto, sem obrigatoriamente se relacionarem com outros agrupamentos. Portanto, a relação entre os sintomas do mesmo agrupamento é mais forte do que a relação com diferentes agrupamentos ${ }^{4}$. Sintomas diferentes podem ocorrer em combinação ou serem influenciados uns pelos outros. A elucidação desses fatores colabora para uma melhor compreensão da fisiologia e do tratamento, culminando com um melhor manejo e controle, direcionando para uma melhor qualidade de vida $(\mathrm{QV})^{5}$.

Quando a doença assume a forma avançada, pode evoluir para a condição de impossibilidade de cura, passando a apresentar sinais e sintomas pouco controláveis, tais como: fadiga, depressão, ansiedade, constipação, dor, náuseas, vômitos, anorexia, entre outros. Nesse sentido, o tratamento oferecido ao paciente toma um viés paliativo em detrimento do curativo, uma vez que essas ocorrências podem estar relacionadas tanto aos efeitos adversos do tratamento quanto à invasáo tumoral e ambos trazem um desconforto ao paciente e um impacto negativo de sua $\mathrm{QV}^{6}$.

Diante disso, os cuidados paliativos têm como função melhorar a QV do paciente ao realizar intervençóes no intuito de aliviar sintomas físicos, psicológicos, sociais e espirituais ${ }^{7}$. Tais aspectos necessitam de atenção em saúde do profissional designado, em virtude dos impactos negativos paralelos à luta incessante do câncer avançado, que podem diminuir a QV do indivíduo se não tratados igualmente ${ }^{8}$.
Existem aproximadamente sete mil serviços de cuidados paliativos em mais de 90 países. Destes, no Brasil, atualmente, são apenas 40 serviços especializados nessa modalidade terapêutica?.

Um estudo realizado em uma Universidade de São Paulo aponta uma possível deficiência na educação de profissionais de saúde no que diz respeito à terminalidade no Brasil. Assim, se faz necessária a mudança de mentalidade dos profissionais que nem sempre estão dispostos e disponíveis para uma nova organização, bem como mudança nos aparelhos formadores que, muitas vezes, dão ênfase na formação técnica em detrimento da humanística, sendo a última extremamente necessária para os cuidados desses pacientes ${ }^{10}$.

De acordo com o Grupo de Estudos sobre Qualidade de Vida da Organização Mundial da Saúde $(\mathrm{OMS})^{11}$, a QV é conceituada como a percepção do indivíduo acerca das influências culturais, sociais, políticas e econômicas no contexto de sua vida. Todos esses aspectos remetem a como ele alcançará seus objetivos, realizando seus sonhos e suprindo suas expectativas, portanto, a QV é considerada subjetiva, refletindo a satisfação do paciente com o curso de sua vida. Não só isso, um projeto colaborativo multicêntrico realizado também pela OMS ${ }^{12}$ expôs que, além da subjetividade, também há uma multidimensionalidade - que inclui ordem física, psicológica, social e espiritual - e uma bipolaridade cotidiana - influências positivas e negativas que impactam na $\mathrm{QV}$.

O objetivo do estudo foi avaliar a relação entre um cluster de sintomas e a qualidade de saúde global de pacientes com câncer avançado.

\section{MÉTODO}

Trata-se de um estudo analítico, transversal, com abordagem quantitativa, realizado em uma instituição hospitalar de natureza filantrópica de médio porte com 150 leitos, localizado no município de Macaé (RJ), Brasil, que atende tanto pacientes pelo Sistema Único de Saúde (SUS), de forma gratuita, como por convênios particulares.

A população do estudo foi composta por pacientes oncológicos em tratamento no hospital do estudo, seguindo os critérios de inclusão: pacientes com câncer em estágio III ou IV, com idade igual ou acima de 18 anos, consciente, lúcido e com capacidade de verbalização para responder aos itens dos instrumentos propostos para o estudo. Os critérios de exclusão foram a impossibilidade de o paciente manter comunicação verbal e/ou escrita.

A amostra calculada foi de 35 pacientes, considerando um nível de significância de $\mathrm{p}<0,05$, com erro tipo I (alfa) esperado de $5 \%$, variável normal padronizada $(Z)$ 
associada ao nível de confiança de 95\%, equivalente a 1,96, em uma população de 160 pacientes em tratamento no hospital do estudo, desvio-padrão (DP) de \pm 15 pacientes, para todas as variáveis.

Utilizou-se a seguinte fórmula para determinar a amostra aleatória simples:

$$
\begin{aligned}
& \mathrm{n}=1,96^{2} \underline{\sigma}^{2} \\
& \varepsilon^{2} \\
& \text { Onde: } \\
& \bullet \mathrm{n}=\text { tamanho da amostra } \\
& \text { - } \sigma=\text { desvio-padrão } \\
& \text { - } \varepsilon=\text { erro }
\end{aligned}
$$

Os dados foram coletados entre julho de 2019 e fevereiro de 2020, por meio de entrevistas, utilizando-se o questionário 30-item European Organization for Research and Treatment of Cancer Core Quality of Life Questionnaire (EORTC QLQ-C30), versão 3. Esse instrumento de coleta foi produzido pela Organização Europeia de Pesquisa e Tratamento do Câncer para uso acadêmico, com o objetivo de avaliar a QV de pacientes com câncer. O questionário possui aplicabilidade validada e está sendo utilizado por diversos estudos no Brasil. O EORTC QLQ-C30 possui um escore de 0-100, sendo que, quanto maior o escore, pior a $\mathrm{QV}$.

Tal instrumento é composto por 30 itens divididos em cinco escalas funcionais (desempenho físico e funcional, função cognitiva, emocional e social), três escalas de sintomas (fadiga, dor e náusea e vômitos), uma escala que avalia a QV em geral, cinco termos únicos (dispneia, distúrbio de sono, perda de apetite, constipação e diarreia) e um item isolado que avalia o impacto financeiro. As respostas são dadas em uma escala do tipo Likert de 4 pontos, com exceção dos itens que avaliam a QV em geral (itens 29 e 30), que utilizam a escala do tipo Likert de 7 pontos.

Os dados foram analisados no software Statistical Package for the Social Sciences (SPSS), versão 21. Para a caracterização dos indivíduos avaliados, as variáveis contínuas foram descritas por média e DP. As variáveis contínuas foram testadas por meio do teste de Kolmogorov-Smirnov para verificar se os dados apresentavam distribuição normal.

Para avaliar a correlação entre a qualidade da saúde global com o escore da escala de sintomas, foi utilizado o coeficiente de correlaçáo de Spearman, uma vez que a amostra não apresentou uma distribuição normal. Sendo utilizada a classificação dos coeficientes de correlação: coeficientes de correlação $<0,4$ (correlação de fraca magnitude), $>0,4 \mathrm{a}<0,5$ (de moderada magnitude) e $>0,5$ (de forte magnitude) ${ }^{13}$.

Realizou-se a análise de regressão linear para estimar a associaçấo entre a variável dependente (qualidade da saúde global) e a escala de sintomas, sendo incluídas nos modelos as 13 perguntas que compóem o escore da escala de sintomas e retidas no modelo final aquelas com p-valor $<0,05$.

O presente estudo foi aprovado pelo Comitê de Ética em Pesquisa do Instituto de Psiquiatria da Universidade Federal do Rio de Janeiro (UFRJ), atendendo às exigências do Conselho Nacional de Saúde sobre as diretrizes e normas para pesquisas com seres humanos, segundo a resolução $n^{\circ}$. 466/2012, sob o número de Parecer: 2.821.570.

\section{RESULTADOS}

Dos pacientes com câncer que participaram do estudo $(\mathrm{n}=146), 140$ (96\%) forneceram dados completos de sintomas e qualidade da saúde global. A média final do escore da escala de sintomas foi igual a 34,7 (DP=26,2) e de $32,3(\mathrm{DP}=25)$ para o escore da qualidade da saúde global.

A idade média dos pacientes foi de 60 anos $( \pm 16,65)$, sendo $57,83 \%$ do sexo feminino, $35,29 \%$ brancos e $64,71 \%$ negros ou pardos (Tabela 1). Os pacientes receberam tratamento/acompanhamento pelo SUS.

Entre as 13 perguntas que compóem o escore de sintomas, destacaram-se quatro sintomas mencionados

Tabela 1. Dados sociodemográficos dos pacientes e tipos de câncer. Macaé, RJ, Brasil, 2020

\begin{tabular}{lll}
\hline Sexo & Feminino & $57,83 \%$ \\
Idade & Masculino & $42,17 \%$ \\
& 80 anos ou mais & $10,74 \%$ \\
& $70-79$ & $18,91 \%$ \\
& $60-69$ & $32,37 \%$ \\
& $50-59$ & $13,46 \%$ \\
& $40-49$ & $10,74 \%$ \\
Cor & $30-39$ & $8,16 \%$ \\
Tipos de câncer & $20-29$ & $5,44 \%$ \\
& Bancos & $35,29 \%$ \\
& Negros ou pardos & $64,71 \%$ \\
& Colorretal & $35,86 \%$ \\
& Próstata & $20,51 \%$ \\
& Estômago & $10,1 \%$ \\
& Pulmão & $7,74 \%$ \\
& Outros & $7,74 \%$ \\
\hline
\end{tabular}

como "não", por mais de $60 \%$ dos avaliados, sendo estes: diarreia $(83,6 \%)$, vômito $(73,6 \%)$, falta de ar $(67,9 \%)$ e enjoos $(60,7 \%)$. Já os sintomas citados com a frequência de "muito", referidos por mais de $20 \%$ dos avaliados, foram: "precisou de descansar" $(28,6)$, "dores perturbaram as suas atividades diárias" (25,7\%), "dores" (26,4\%), "sentiu-se fraco" (24,3\%) e "sentiu-se cansado" (20\%) (Tabela 2). 
Tabela 2. Perguntas que abrangeram o escore de sintomas, $n=140$. Macaé, RJ, Brasil, 2020

\begin{tabular}{|c|c|c|c|c|c|}
\hline \multirow{2}{*}{ Perguntas } & \multirow{2}{*}{$\begin{array}{l}\text { Média do } \\
\text { escore (DP) }\end{array}$} & \multicolumn{4}{|c|}{$\begin{array}{l}\text { Escala de sintomas } \\
\qquad \mathrm{n}(\%)\end{array}$} \\
\hline & & $\begin{array}{c}\text { Não } \\
\text { (1 ponto) }\end{array}$ & $\begin{array}{l}\text { Um pouco } \\
\text { ( } 2 \text { pontos) }\end{array}$ & $\begin{array}{l}\text { Bastante } \\
\text { (3 pontos) }\end{array}$ & $\begin{array}{c}\text { Muito } \\
\text { (4 pontos) }\end{array}$ \\
\hline Precisou de descansar? & $2,33(1,2)$ & $44(31,4)$ & $46(32,9)$ & $10(7,1)$ & $40(28,6)$ \\
\hline Sentiu-se fraco/a? & $2,17(1,2)$ & $58(41,4)$ & $34(24,3)$ & $14(10)$ & $34(24,3)$ \\
\hline Sentiu-se cansado/a? & $2,12(1,1)$ & $58(41,4)$ & $35(25)$ & $19(13,6)$ & $28(20)$ \\
\hline Teve enjoos? & $1,75(1,0)$ & $85(60,7)$ & $25(17,9)$ & $10(7,1)$ & $20(14,3)$ \\
\hline Vomitou? & $1,51(0,9)$ & $103(73,6)$ & $19(13,6)$ & $2(1,4)$ & $16(11,4)$ \\
\hline Teve dores? & $2,24(1,2)$ & $54(38,6)$ & $35(25)$ & $14(10)$ & $37(26,4)$ \\
\hline $\begin{array}{l}\text { As dores perturbaram as } \\
\text { suas atividades diárias? }\end{array}$ & $1,14(1,2)$ & $67(47,9)$ & $23(16,4)$ & $14(10)$ & $36(25,7)$ \\
\hline Teve falta de ar? & $1,64(1,0)$ & $95(67,9)$ & $19(13,6)$ & $8(5,7)$ & $18(12,9)$ \\
\hline Teve dificuldade em dormir? & $1,93(1,1)$ & $72(51,4)$ & $28(20)$ & $18(12,9)$ & $22(15,7)$ \\
\hline Teve falta de apetite? & $1,86(1,1)$ & $82(58,6)$ & $21(15)$ & $12(8,6)$ & $25(17,9)$ \\
\hline Teve prisão de ventre? & $1,81(1,1)$ & $83(59,3)$ & $20(14,3)$ & $17(12,1)$ & $20(14,3)$ \\
\hline Teve diarreia? & $1,29(0,7)$ & $117(83,6)$ & $12(8,6)$ & $4(2,9)$ & $7(5)$ \\
\hline $\begin{array}{l}\text { O seu estado físico ou } \\
\text { tratamento médico } \\
\text { causaram-lhe problemas de } \\
\text { ordem financeira? }\end{array}$ & $1,83(1,0)$ & $75(53,6)$ & $31(22,1)$ & $17(12,1)$ & $17(12,1)$ \\
\hline
\end{tabular}

Para as duas perguntas que abarcam o escore da qualidade da saúde global e apresentam variação entre 1 (péssima) a 7 (ótima) pontos, a média final foi de 3 $(\mathrm{DP}=1,6)$ para a pergunta "Como classificaria a sua saúde em geral durante a última semana?” e de 2,87 $(\mathrm{DP}=1,6)$ para "Como classificaria a sua qualidade de vida global durante a última semana?”. Destacando-se que $84,3 \%$ dos avaliados relataram pontuaçấo $\leq 4$ para ambas as perguntas.

A correlação de Spearman mostrou que há uma correlaçáo positiva e forte entre o escore final da escala de sintomas e o escore final da avaliação global da saúde $(\rho=0,605 ; p<0,001)$.

Foi desenvolvido um modelo de regressão linear múltipla para avaliar a associação entre os sintomas referidos pelos pacientes com câncer e a qualidade da saúde global. O modelo final para qualidade da saúde global incluiu três perguntas sobre o escore dos sintomas: "Precisou descansar?", "Sentiu-se cansado/a?" e "Teve falta de ar?". Observou-se que o incremento de 1 ponto na pergunta "Precisou descansar?" associou-se com o aumento de 5,87 pontos no escore da qualidade da saúde global $(\mathrm{p}<0,01)$; para a pergunta "Sentiu-se cansado/a?", o aumento foi de 6,14 pontos ( $<<0,01$ ); já para "Teve falta de ar?", o aumento foi de 5,08 pontos $(\mathrm{p}<0,01)$ (Tabela 3)
Tabela 3. Associação entre os sintomas referidos e a qualidade da saúde em pacientes com câncer $(n=140)$, avaliada em modelos de regressão linear múltipla. Macaé, RJ, Brasil, 2020

\begin{tabular}{|c|c|c|}
\hline & \multicolumn{2}{|c|}{$\begin{array}{l}\text { Qualidade da saúde } \\
\text { global }^{*}\end{array}$} \\
\hline & $\begin{array}{l}\text { Coeficiente de } \\
\text { regressão } \\
\text { (IC 95\%) }\end{array}$ & p-valor \\
\hline Precisou descansar? & $5,87(2,07 ; 9,68)$ & $<0,01$ \\
\hline Sentiu-se cansado/a? & $6,14(2,09 ; 10,2)$ & $<0,01$ \\
\hline Teve falta de ar? & $5,08(1,32 ; 8,83)$ & $<0,01$ \\
\hline
\end{tabular}

$\left(^{*}\right)$ Estimado pelo EORTC QLQ-C3014.

\section{DISCUSSÃO}

A avaliação de clusters é uma ferramenta que ajuda a diminuir as imprecisôes introduzidas pela avaliação não agrupada dos sintomas. Portanto, o reconhecimento da importância dos agrupamentos pode mudar a prática clínica e permitir o aprimoramento de intervençóes, interferindo diretamente no cuidado ao paciente. $\mathrm{O}$ presente estudo apresentou evidências estatísticas de forte correlação positiva entre um cluster de sintomas e a saúde global de pacientes com câncer avançado. 
O paciente com câncer avançado experimenta um conjunto de sintomas que agridem intensamente o seu bem-estar. O estágio é um algarismo romano de I (1) a IV (4) para a maioria dos cânceres. Os cânceres em estágio I são menos avançados e costumam ter um melhor prognóstico. Já os cânceres em estágios mais elevados geralmente têm mais características preocupantes, como metástases, entấo podem exigir um tratamento mais intenso. Alguns tipos de câncer também têm estágio 0 , o qual significa que o câncer ainda está apenas na camada de células onde começou, e não se espalhou mais ${ }^{15}$.

Como os estágios III e IV do câncer são os mais avançados, exigem complexibilidade na assistência, sendo os cuidados paliativos um grande aliado. Dessa maneira, os cuidados paliativos são de responsabilidade de uma equipe multidisciplinar, que deve ter preparo para lidar com os medos, sofrimentos e angústias do paciente e sua família, sabendo como agir frente à realidade da finitude humana e às necessidades do paciente ${ }^{16}$.

Nesse sentido, quanto maior o número de sintomas relatados por um paciente em cuidados paliativos, menor será sua QV, sendo que os clusters de sintomas mais recorrentes são: dor, fadiga, perda de apetite, vômitos, perda de peso, constipação, palidez, caquexia, dispneia, baixa qualidade de sono, disfagia, depressão, disgeusia, baixo estado nutricional, sensação de dependência e falta de apoio familiar e social ${ }^{17}$.

Entre os sintomas supracitados, a dor do câncer é relatada em $70 \%$ a $90 \%$ dos casos avançados, caracterizada como um sintoma severo e um dos principais indicados pelos pacientes ${ }^{18}$. O Brasil tem se apresentado como o país com o maior índice de queixas de dor oncológica da América Latina, afetando de $25 \%$ a $30 \%$ dos pacientes na fase inicial, $50 \%$ em estágios variados do câncer e de $70 \%$ a $90 \%$ daqueles no estágio avançado ${ }^{19}$. Nesse sentido, o alívio da dor é um dos principais objetivos cuidados paliativos, visto que acarreta sofrimento e até incapacidade de realização de atividades diárias, assim, tem-se um desafio para os profissionais de saúde designados a tratá-la, uma vez que a dor é um sintoma subjetivo que ocorre quando o paciente diz ter e existe e quando ele diz existir. Assim, requer uma capacitação dos profissionais para que se tenha eficácia no tratamento ${ }^{20}$.

Entre o cluster formado na presente pesquisa, três sintomas se destacaram durante a coleta de dados: cansaço, fadiga e dispneia. Todos apresentaram um aumento de escore na qualidade de saúde global quando obtinham aumento de um ponto nas perguntas referentes a esses sintomas, demonstrando a necessidade de atenção a esses três itens.

Sabendo-se que os clusters de sintomas são definidos como grupos de pelo menos dois ou três sintomas simultâneos que estão relacionados entre $\mathrm{s}^{21}$, enfermeiras brasileiras realizaram uma revisão sistemática e destacaram que os sintomas agrupados mais encontrados são os neuropsicológicos e os gastrointestinais ${ }^{22}$.

Um estudo norte-americano observou que a dor, a dispneia, a fadiga e o estresse emocional surgem em simultâneo e são interdependentes, logo, apresentam uma inter-relação entre si, tendo em conta que podem produzir efeito acumulativo ${ }^{23}$. Já em outro estudo brasileiro, foi utilizada a escala de sintomas do EORTC QLQ-C30, na qual dor, fadiga, insônia e perda do apetite tiveram destaque juntos ${ }^{24}$.

O sono de má qualidade esteve presente em cerca de $75 \%$ dos participantes deste estudo, corroborando as literaturas internacional e nacional, em que autores alertam para os prejuízos do sono na pessoa com câncer ${ }^{25}$. Nos pacientes com sono de má qualidade, os problemas de sono constituíram clusters com os sintomas de preocupação e tristeza.

Um estudo sobre agrupamentos de pacientes com câncer de pulmão fez a análise de clusters e levou em conta a magnitude dos sintomas de maior prevalência de acordo com as escalas de sintomas do EORTC QLQC-30; esses sintomas foram fadiga, dor, dispneia e insônia. Entre os resultados, foram identificados três agrupamentos (subgrupos) de pacientes, baseados na magnitude dos quatro sintomas mais prevalentes. Os três subgrupos de pacientes foram os seguintes: pacientes com sintomas leves $(\mathrm{n}=30 ; 60 \%)$; pacientes com sintomas moderados $(\mathrm{n}=14$; $28 \%)$ e pacientes com sintomas graves $(n=6 ; 12 \%)$. O subgrupo de pacientes com sintomas graves apresentou a pior QV, conforme mensurada pelos escores totais e pelas dimensôes integradas dos três instrumentos ${ }^{26}$.

No cluster formado pela presente pesquisa, a fadiga e o cansaço estavam presentes. A fadiga pode ser descrita como um sintoma de desequilíbrio entre o estado de descanso e de atividade, o que ocasiona uma falta de energia tanto física quanto mental, porém o indivíduo que a relata se recupera ao cessar a causa do desgaste ${ }^{27}$. A fadiga relacionada ao câncer (FRC) é diferente desse quadro, em virtude de não haver alívio do sintoma com sono/ repouso, por isso é considerada um fator de diminuição da QV e, consequentemente, da satisfação pessoal. Os pacientes em tratamento oncológico, principalmente os submetidos à quimioterapia (QT), demonstram a persistência do sintoma após o término do tratamento e até mesmo da recuperação da doença. Esse prolongamento da fadiga a faz ser caracterizada como crônica e implica na possibilidade de uma adaptação metabólica e fisiológica, como descondicionamento e caquexia, por exemplo ${ }^{28}$.

Muitos pacientes com câncer desenvolvem anemia como consequência de sua doença maligna, tratamento ou 
até mesmo por comorbidades previamente apresentadas. A anemia é uma das maiores causas reversíveis de FRC ${ }^{29}$, estando presente em mais de $40 \%$ dos casos, e, em pacientes durante a QT, a incidência pode elevar-se a $90 \%{ }^{30}$.

Outro sintoma que demonstrou destaque nesta pesquisa foi a dispneia. Tal sintoma pode correlacionar-se com a diminuição dos níveis de hemoglobina, o que impacta negativamente a sobrevida e acentua a FRC ${ }^{31}$. O termo dispneia é utilizado para definir uma sensação subjetiva de falta de ar ou dificuldade respiratória que o doente diz sentir, sendo um dos sintomas mais prevalentes em diversos estudos, ocorrendo entre $19 \%$ e $51 \%$ dos doentes oncológicos, e rapidamente aumenta com a progressão da doença e no fim de vida, especialmente em doentes com câncer de pulmão ${ }^{32}$.

Este estudo apresenta limitaçôes, tais como a amostra ser de conveniência e relativamente pequena, fato este que se atribui à realização em uma cidade do interior do Estado do Rio de Janeiro, o que impediu a análise do efeito do cluster de sintomas estudado sobre a qualidade de saúde global de pacientes com câncer, considerando a influência de outras variáveis, como sexo, idade, escolaridade. Assim, sugere-se que novos estudos sejam realizados, a fim de avaliar o impacto da saúde global de pacientes com câncer, bem como a possível modificação de efeitos ao considerar outros clusters de sintomas.

Para cuidar do paciente com câncer avançado e, muitas vezes, fora da possibilidade de cura atual, é necessário pensar de forma multidisciplinar e, até mesmo, interdisciplinar. Uma equipe de cuidados paliativos tem, em sua essência, a multidisciplinaridade. Essa necessidade se deve à heterogeneidade das necessidades individuais dos pacientes e familiares. Nesse contexto, nenhuma formação profissional é suficientemente ampla para abordar todos os aspectos que precisam de atençáo ${ }^{33}$. Sendo assim, para atingir a qualidade de saúde global, é preciso refletir sobre o cuidado de forma integral para atender a todas as dimensóes do cuidado, quais sejam, biopsicossocioespirituais.

\section{CONCLUSÃO}

O presente estudo, realizado em uma cidade do interior do Estado do Rio de Janeiro, demonstrou que existe uma correlação positiva forte entre o cluster de sintomas composto por dor, fadiga, dispneia, náusea, vômito, insônia, inapetência, constipaçấo, diarreia e a qualidade de saúde global de pacientes com câncer avançado. Logo, sugere-se a possibilidade de sua aplicabilidade em outros cenários do país como, por exemplo, em grandes centros urbanos.

\section{CONTRIBUIÇÕES}

Todos os autores contribuíram na concepção e/ ou no planejamento do estudo; na obtenção, análise e interpretação dos dados; assim como na redação e revisão crítica; e aprovaram a versão final a ser publicada.

\section{DECLARAÇÃO DE CONFLITO DE INTERESSES}

Nada a declarar.

\section{FONTES DE FINANCIAMENTO}

Não há.

\section{REFERÊNCIAS}

1. Bray F, Ferlay J, Soerjomataram I, et al. Global cancer statistics 2018: GLOBOCAN estimates of incidence and mortality worldwide for 36 cancers in 185 countries. CA Cancer J Clin. 2018;68(6): 394-424. doi: http://doi.org/ $10.3322 /$ caac. 21492

2. Instituto Nacional de Câncer José Alencar Gomes da Silva. Estimativa 2020: incidência de câncer no Brasil [Internet]. Rio de Janeiro: INCA; 2019 [acesso 2020 maio 15]. Disponível em: https://www.inca.gov.br/sites/ ufu.sti.inca.local/files/media/document/estimativa-2020incidencia-de-cancer-no-brasil.pdf

3. Lopes-Júnior LC, Bomfim EO, Nascimento LC, et al. Teoria dos sintomas desagradáveis: subsídios para o manejo de sintomas em crianças e adolescentes oncológicos. Rev Gaúcha Enferm. 2015;36(3):109-12. doi: https://doi.org/10.1590/1983-1447.2015.03.51465

4. Carrillo-González GGM. Los grupos de síntomas en personas con cáncer: una revisión integrativa. Aquichan. 2017;17(3):257-69. doi: http://doi.org/10.5294/ aqui.2017.17.3.3

5. Delalibera M, Presa J, Barbosa A, et al. Sobrecarga no cuidar e suas repercussóes nos cuidadores de pacientes em fim de vida: revisão sistemática da literatura. Ciênc Saúde Coletiva. 2015;20(9):2731-47. doi: https://doi. org/10.1590/1413-81232015209.09562014

6. Leite MAC, Nogueira DA, Terra FS. Social and clinical aspects of oncological patients of a chemotherapy service. Rev Rene. 2015;16(1):38-45. doi: https://doi. org/10.15253/2175-6783.2015000100006

7. Hermes HR, Lamarca ICA. Cuidados paliativos: uma abordagem a partir das categorias profissionais de saúde. Ciênc Saúde Coletiva. 2013;18(9):2577-88. doi: https:// doi.org/10.1590/S1413-81232013000900012

8. Mansano STC, Ceolim MF. Qualidade de vida de pacientes com câncer no período de quimioterapia. Texto Contexto - Enferm. 2012;21(3):600-7. doi: https://doi. org/10.1590/S0104-07072012000300015 
9. Cardoso DH, Muniz RM, Schwartz E, et al. Cuidados paliativos na assistência hospitalar: a vivência de uma equipe multiprofissional. Texto Contexto - Enferm. 2013;22(4):1134-41. doi: https://doi.org/10.1590/ S0104-07072013000400032

10. Kovács MJ. Educação para a morte. Psicol Cienc Prof. 2005;25(3):484-97. doi: http://doi.org/10.1590/S141498932005000300012

11. Meneguin S, Matos TDS, Ferreira MLSM. Percepção de pacientes oncológicos em cuidados paliativos sobre qualidade de vida. Rev Bras Enferm. 2018;71(4):19982004. doi: https://doi.org/10.1590/0034-7167-20170360

12. The World Health Organization Quality of Life assessment (WHOQOL): position paper from the World Health Organization. Soc Sci Med. 1995;41(10):14039. doi: http://doi.org/10.1016/0277-9536(95)00112-k

13. Hulley SB, Cummings SR, Browner WS, et al. Delineando a pesquisa clínica: uma abordagem epidemiológica. 2. ed. Porto Alegre: Editora Artmed; 2003.

14. Fayers PM, Aaronson NK, Bjordal K, et al. EORTC QLQ-C30: scoring manual. 3rd ed. Brussels: European Organization for Research and Treatment of Cancer; 2001.

15. American Cancer Society [Internet]. Atlanta (GA): American Cancer Society; c2020. Cancer Staging; [revised 2020 June 18; cited 2020 Dec 6]. Available from: https://www.cancer.org/treatment/understanding-yourdiagnosis/staging.html

16. Machado KDG, Pessini L, Hossne WS. A formação em cuidados paliativos da equipe que atua em unidade de terapia intensiva: um olhar da bioética. Rev Bioethikos [Internet]. 2007 [acesso $2020 \mathrm{dez}$ 09];1(1):34-42. Disponível em: http://www.saocamilo-sp.br/pdf/ bioethikos/54/A_cuidados_paliativos.pdf

17. Matsumoto DY. Cuidados paliativos: conceito, fundamentos e princípios. In: Carvalho RT, Parsons HA, organizadores. Manual de cuidados paliativos ANCP. 2. ed. Rio de Janeiro: Academia Nacional de Cuidados Paliativos; 2012. p. 23-30.

18. Cunha FF, Rêgo LP. Enfermagem diante da dor oncológica. Rev Dor. 2015;16(2):142-5. doi: https:// doi.org/10.5935/1806-0013.20150027

19. Oliveira AL, Palma Sobrinho N, Cunha BAS. Manuseio da dor crônica em pacientes oncológicos pela equipe de enfermagem. Rev Dor. 2016;17(3):219-22. doi: https:// doi.org/10.5935/1806-0013.20160075

20. World Health Organization [Internet]. Geneva: WHO; c2021. Palliative care; [date unknown] [cited 2020 Mar 15]. Available from: http://www.who.int/cancer/ palliative/definition/en

21. Wittmann-Vieira R, Goldim JR. Bioética e cuidados paliativos: tomada de decisôes e qualidade de vida.
Acta Paul Enferm. 2012;25(3):334-9. doi: https://doi. org/10.1590/S0103-21002012000300003

22. Boeira SF, Guimarães RM, Acioli LR, et al. Cluster de sintomas e câncer na pesquisa em enfermagem: revisão sistemática. Rev Bras Cancerol. 2014;60(4):3516. doi: https://doi.org/10.32635/2176-9745. RBC.2014v60n4.462

23. Cleeland CS, Sloan JA; ASCPRO Organizing Group. Assessing the Symptoms of Cancer Using PatientReported Outcomes (ASCPRO): searching for standards. J Pain Symptom Manage. 2010;39(6):1077-85. doi: https://doi.org/10.1016/j.jpainsymman.2009.05.025

24. Freire MEM, Costa SFG, Lima RAG, et al. Qualidade de vida relacionada à saúde de pacientes com câncer em cuidados paliativos. Texto Contexto Enferm. 2018;27(2):e5420016. doi: https://doi. org/10.1590/0104-070720180005420016

25. Nunes NAH, Ceolim MF. Qualidade do sono e cluster de sintomas em pacientes com câncer em tratamento quimioterápico. Cogitare Enferm. 2019;24:e58046. doi: http://doi.org/10.5380/ce.v24i0.58046

26. Franceschini J, Jardim JR, Fernandes ALG, et al. Relação entre a magnitude de sintomas e a qualidade de vida: análise de agrupamentos de pacientes com câncer de pulmão no Brasil. J Bras Pneumol. 2013;39(1):23-31. doi: http://doi.org/10.1590/S1806-37132013000100004

27. Anjos ACY, Campos CS, Cunha NF, et al. Fadiga secundária à quimioterapia em mulheres com câncer de mama: revisão integrativa de literatura. Rev Perspect Psicol. 2017;21(2):47-70. doi: https://doi.org/10.14393/ PPv21n2a2017-05

28. Borges JA, Quintão MMP, Chermont SSMC, et al. Fadiga: um sintoma complexo e seu impacto no câncer e na insuficiência cardíaca. Int J Cardiovasc Sci. 2018;31(4):433-42. doi: http://doi.org/10.5935/23594802.20180027

29. Campos MPO, Hassan BJ, Riechelmann R, et al. Fadiga relacionada ao câncer: uma revisão. Rev Assoc Med Bras. 2011;57(2):211-9. doi: https://doi.org/10.1590/S010442302011000200021

30. Dicato M, Plawny L, Diederich, M. Anemia in cancer. Ann Oncol. 2010;21(Suppl 7):VII167-VII172. doi: https://doi.org/10.1093/annonc/mdq284

31. Calabrich AFC, Katz A. Deficiência de ferro no paciente com câncer. Rev Bras Hematol Hemoter. 2010;32(Supl 2):95-8. doi: https://doi.org/10.1590/ S1516-84842010005000049

32. Viola R, Kiteley C, Lloyd NS, et al. The management of dyspnea in cancer patients: a systematic review. Support Care Cancer. 2008;16(4):329-37. doi: https://doi. org/10.1007/s00520-007-0389-6

33. Higginson IJ, Evans CJ. What is the evidence that palliative care teams improve outcomes for cancer 
Mello IR, Guimarães NML, Monteiro LS, Taets GCC

patients and their families? Cancer J. 2010;16(5):423-35.

doi: https://doi.org/10.1097/PPO.0b013e3181f684e5

Recebido em 5/8/2020

Aprovado em 10/12/2020 